\title{
New North American Isolates of Venturia inaequalis Can Overcome Apple Scab Resistance of Malus floribunda 821
}

\author{
David Papp, Jugpreet Singh, David Gadoury, and Awais Khan ${ }^{\dagger}$ \\ ${ }^{1}$ Plant Pathology and Plant-Microbe Biology Section, Cornell University, Geneva, NY 14456, U.S.A.
}

\begin{abstract}
Apple scab, caused by Venturia inaequalis, is a destructive fungal disease of major apple cultivars worldwide, most of which are moderately to highly susceptible. Thus, development of scab resistant cultivars is one of the highest priorities of apple breeding programs. The principal source of resistance for breeding programs has been the scab resistance gene Rvi6 that originated from the Japanese crabapple Malus floribunda (Sieb.) sel. 821. Isolates of $V$. inaequalis able to overcome Rvi6 have been identified in Europe, but have not yet been reported on the American continents. We recently discovered scab infection on $M$. floribunda 821 trees in a research orchard at Geneva, NY, U.S.A., where approximately $10 \%$ of the leaves bore profusely sporulating apple scab lesions,

isolates in total, of North American and European origin, isolated from M. floribunda 821, 'Nova Easygro', 'Golden Delicious', TSR33T239, 'Schone van Boskoop', and 'Prima', using 16,321 genome-wide SNPs. Population genetic structure and PCA separated the isolates into distinct European and U.S. groups. The forgoing suggests that the new Rvi6 virulent isolates emerged within U.S. populations, rather than being transported from Europe. The complete resistance breakdown in $M$. floribunda 821 but not in descendant cultivars, which kept their field resistance, suggests that durable resistance to apple scab will require a more comprehensive understanding of Rvi6 mediated resistance in diverse genetic backgrounds.
\end{abstract} many of which had coalesced to cover entire leaves. We observed both chlorosis, typical to Rvi6, and pinpoint pitting symptoms typical to failed infections by $V$. inaequalis on hosts bearing the $R v i 7$ gene. We assessed genetic diversity and population genetic structure of $11 \mathrm{~V}$. inaequalis
Keywords: Population genetic structure, virulence, pathogenicity, genome sequencing, disease management, cultivar/resistance, fungi, fruit, tree fruits
Apple scab, caused by the Ascomycete fungus Venturia inaequalis (Cke.) Wint., is the economically most important fungal disease of apples. In nearly all apple growing regions of the world, growers rely on fungicide applications each year to limit fruit quality and yield losses due to scab. The decreasing susceptibility of $V$. inaequalis populations to fungicides has led to a need for alternative pest control strategies. Distressingly, current IPM strategies could be counterproductive by expressing heavy selection pressure on fungicide resistance (Beckerman et al. 2015). Recently, $V$. inaequalis isolates were reported to have resistance toward multiple fungicides without having been affected by significant fitness cost (Chapman et al. 2011). Scab-resistant cultivars require fewer fungicide applications, and hence are of high importance to organic production systems and home gardens (Brown and Maloney 2008). However, the commercial success of scab-resistant cultivars has been limited because of their generally low fruit quality. The need to control scab without total reliance upon fungicides has therefore made development of scab resistant cultivars with high fruit quality one of the highest priorities of apple breeding programs.

${ }^{\dagger}$ Corresponding author: A. Khan; awais.khan@cornell.edu

Funding: This research was supported by the New York Apple Research and Development Program (ARDP). David Papp is supported by the Miklos Faust scholarship in fruit science.

*The $\boldsymbol{e}$-Xtra logo stands for "electronic extra" and indicates that one supplementary figure is published online.

The author(s) declare no conflict of interest.

Accepted for publication 29 November 2019

C 2020 The American Phytopathological Society
The vast majority of the modern scab resistant cultivars derive from a Japanese crabapple clone, Malus floribunda (Sieb.) sel. 821 , which was found to be completely free of scab in the beginning of the twentieth century. At the University of Illinois, C. S. Crandall built a collection of crabapples and, among other hybridizations, crossed two siblings from a M. floribunda $821 \times$ 'Rome Beauty' progeny (Crandall 1926). Two accessions from this cross progeny were later selected for their exceptional scab resistance yet large fruit size, and were used as resistance donors by the PRI (Purdue University, Rutgers University, and the University of Illinois) cooperative breeding program. The first PRI cultivar, 'Prima' was released in 1967, and since then several breeding centers have developed resistant cultivars by using PRI materials (Crosby et al. 1992). Currently, there are more than one hundred modern scab resistant apple cultivars, with approximately $90 \%$ of them carrying the resistance derived from M. floribunda 821 (Brown and Maloney 2008, 2013).

To date, $18 \mathrm{~V}$. inaequalis resistance genes (Rvi genes) including Rvi6 and Rvi7 from M. floribunda 821 and genes from other Malus accessions have been described. Gene-for-gene relationships are assumed for the majority of them (Broggini et al. 2011; Bus et al. 2011; Khajuria et al. 2018). Based on the segregation, the resistance of $M$. floribunda 821 was also thought to rely on a single dominant gene, previously called $V f$ (syn. Rvi6), exhibiting resistance symptoms from hypersensitive reaction to chlorosis (occasionally necrosis or slight sporulation). However, segregation analysis of the resistance symptoms suggested the presence of an additional gene for scab resistance (Parisi et al. 1993; Williams and Kuc 1969). A large-scale pathogenicity study on a 'Golden Delicious' $\times$ M. floribunda 821 progeny demonstrated the presence of the $V f h$ (syn. Rvi7) gene responsible for the hypersensitive reaction in M. floribunda 821, which was distinct from the original Rvi6 (Bénaouf and Parisi 2000). The $V f h$ gene has been lost early on in the breeding process, or has been overcome by the pathogen (Bénaouf and Parisi 2000; Gessler 1989; Parisi and Lespinasse 1996). 
Although the resistance conferred by a single major gene can be easily inherited, as preferred by breeders, the breakdown of major genes as a result of the fast evolution of a pathogen has been documented many times in several host-pathogen systems (Pink and Hand 2003). For instance, chlorosis with slight sporulation was observed on 'Prima' (Rvi6) in the greenhouse trials even before its release, whereas Rvi6 cultivars remained resistant under field conditions. Thus, the resistance conferred by Rvi6 presents as partial resistance when the environmental factors are optimal for disease development, although the 1:1 segregation ratio of resistance expected from a single major gene was only observed if progeny expressing necrotic, slightly sporulating symptoms were still classified resistant (Crosby et al. 1992; MacHardy 1996). It has been demonstrated that the deviation from the expected segregation ratio is caused by sublethal genes ( $s l l$ and/or $s l 2$ in case of the presence of a third gene $s l 3$ ) that are linked to the Rvi6 region (Gao and Van De Weg 2006). In Europe (Ahrensburg, Germany), sporulating lesions were detected in the field on 'Prima' in 1984, and in 1988 on 'Prima' and 'Coop' 7, 9, and 10 in the same orchard, although 'Prima' and 'Coop' 7, 9, and 10 were all resistant in the greenhouse. The inoculum from Ahrensburg and monoconidial isolates were used for testing various Rvi6 cultivars/selections, all of which were susceptible to the new isolates, but the original source of the resistance, $M$. floribunda 821, remained intact (Parisi et al. 1993). The breakdown of the resistance of M. floribunda 821 (carrying both Rvi6 and Rvi7) was reported later in East Malling, England (Roberts and Crute 1994). Interestingly, the isolates overcoming the resistance of $M$. floribunda 821 were avirulent on 'Golden Delicious' and on its resistant descendants (e.g., 'Prima' and 'Florina') harboring the ephemeral Rvil gene. However, it is still unclear whether the isolates were unable to overcome the resistance of these cultivars due to the Rvil gene, or other genetic factors prohibited the adaptation of the pathogen (Gessler and Pertot 2012; Roberts and Crute 1994). It was reported that the background resistance of M. floribunda 821 is low, and its severe infection happens when the two major genes Rvi6 and Rvi7 have been overcome. This also suggests that the $R v i 6$ resistant cultivars infected by less aggressive isolates might possess more complex resistance than those inherited from M. floribunda 821 (Caffier et al. 2010; Parisi et al. 2004).

Further population genetic studies suggested that European Rvi6virulent isolates did not emerge from a recent mutation in the AvrRvi6 gene, but have pre-existed for thousands of years in wild crabapple reservoirs, without relevant gene-flow to populations infecting domesticated apples. The virulent isolates on Rvi6 cultivars are genetically less variable, but distinct from those collected from susceptible cultivars, suggesting a genetic bottleneck in their recent history (Guérin and Le Cam 2004; Guérin et al. 2007; Lemaire et al. 2016; Michalecka et al. 2018). Although the Rvi6 virulent and nonvirulent lineages might have been separated for thousands of years, the emergence of Rvi6 cultivars in Europe allowed gene flow between the two lineages, which might have increased the speed of the pathogen's adaptation, especially in orchards where susceptible and resistant cultivars coexist (Michalecka et al. 2018). Human facilitated transport might represent another factor for the spread of Rvi6 virulent strains, and a possible cause of the inconsistency between the geographical origin and genetic polymorphism of isolates in several studies (Gladieux et al. 2010; Guérin et al. 2007; Kaymak et al. 2016; Michalecka et al. 2018).

New isolates that can overcome the resistance of $M$. floribunda 821 have not yet been reported on the American continent, but the breakdown of the resistance was presumed by Beckerman from 2007, after seeing scab symptoms on $M$. floribunda 821 , but never confirmed. Later in 2008 and 2009, scab-like symptoms were documented on 'Pristine' ( $V f)$ and other $V f$ accessions ('Pixie Crunch', 'Jonafree', 'Enterprise') (https://www.extension.purdue.edu/extmedia/ BP/BP-76-W.pdf). To monitor the race comparison of $V$. inaequalis in North America, a 33-year-long assay was carried out in the Secrest Arboretum (Ohio), where heavy sporulation was observed on a Japanese crabapple tree in 2003 and 2005, although its resistance was effective for decades previously. The authors, however, did not confirm whether the accession was identical to M. floribunda 821 (Beckerman et al. 2009). In this study, we report new $V$. inaequalis isolates collected from the infected $M$. floribunda 821 trees grown in an apple orchard, their origin and genetic diversity, and their pathogenicity on Rvi6 resistant 'Macfree'.

\section{Materials and Methods}

Field assessment of apple scab symptoms. In June 2019, apple scab infection was observed on approximately 20-year-old trees of M. floribunda 821 and scab resistant apple cultivars 'Prima' and 'Nova Easygro' grown in the research orchard Darrow Farm (Geneva, U.S.A.). In addition, four Rvi6 resistant PRI cultivars, 'Dayton', 'Jonafree', 'Priscilla', 'Redfree', as well as 'Gala', 'Macintosh', 'Golden Delicious', and the French resistant cultivar 'Florina' were present within the same orchard. The trees from each genotype were present in four replications in a randomly distributed pattern across the entire orchard space. The orchard was maintained without any fungicide spray since 2017 (Table 1).

Apple scab incidence was assessed using the 0-9 grade scale of Lateur and Populer (1994) as follows: 0 - no observation (missing plant), 1 - no visible lesions, 2 - one or very few lesions detectable on close scrutiny of the tree ( 0 to $1 \%), 3$ - immediately apparent lesions in general clustered in few parts of the tree (1 to 5\%), 4 - intermediate, 5 - numerous lesions widespread over a large part of the tree $( \pm 25 \%)$, 6 - intermediate, 7 - severe infection with half of the leaves badly infected by multiple lesions $( \pm 50 \%), 8$ - intermediate $( \pm 75 \%)$, or 9 - tree completely affected with (nearly) all the leaves badly infected by multiple lesions ( $>90 \%)$. Reaction type was determined according to the scale used by the PRI breeding program (Crosby et al. 1992) as follows: 0 - no symptom, 1 - pin point pits, 2 - irregular chlorotic or necrotic lesions, 3 - few restricted sporulation (M) mixture of necrotic and chlorotic and sparsely sporulating lesions, or 4 abundant sporulation.

Sample collection. Scab infected leaves were collected from M. floribunda 821 'Nova Easygro' trees located in Darrow Farm (Geneva, NY) (Table 2). Monospore isolates were prepared by sticking infected leaves to the inner top side of petri dishes containing $1.2 \%$

Table 1. Apple scab symptoms and incidence on Rvi6 cultivars and susceptible controls. Note: PRI stands for Purdue, Rutgers, Illinois apple breeding program.

\begin{tabular}{|c|c|c|c|c|c|}
\hline PI number & Cultivar name & $R v i$ genes & Symptom class $(0-4)$ & Incidence (0-9) & Source \\
\hline 392303 & Gala & None & 4 & 5 & - \\
\hline 590186 & Wijcik McIntosh & None & 4 & 8 & - \\
\hline 588998 & Marshall McIntosh & None & 4 & 8 & - \\
\hline 590184 & Golden Delicious & Rvil & 4 & 7 & - \\
\hline 589827 & M. floribunda 821 & Rvi6, Rvi7 & 4 & 4 & - \\
\hline 588747 & Florina & Rvi1, Rvi6 & 0 & 1 & EU (French) \\
\hline 588838 & Nova Easygro & Rvi6 & M & 2 & PRI (Canadian) \\
\hline 589181 & Prima & Rvil, Rvi6 & 3 & 2 & PRI \\
\hline 589962 & Jonafree & Rvi6 & 0 & 1 & PRI \\
\hline 590183 & Dayton & Rvi6 & 0 & 1 & PRI \\
\hline 594111 & Redfree & Rvi6 & 0 & 1 & PRI \\
\hline 589965 & Priscilla & Rvi6 & 0 & 1 & PRI \\
\hline
\end{tabular}


water agar. The dishes were sealed and incubated overnight to let the spores fall on the agar and germinate. Single germinating spores were observed under a microscope and were carefully transferred to new PDA plates under sterile conditions to avoid the transfer of any contaminant. Scab isolates 'VI-1797-9' and 'VI-NY05-GD' were previously collected from another orchard in Geneva, NY, and TSR33T239 (carrying Rvi4) was collected from Ohio, U.S.A. (Schnabel et al. 1999). The well-characterized reference $V$. inaequalis isolates of European origin EU-B05 and EU-NL24 (Caffier et al. 2015) were obtained from INRA, France.

Artificial inoculation of the Rvi6 resistant commercial cultivar 'Macfree'. Conidial suspension was prepared by collecting abundantly sporulating leaves from the field (Darrow Farm), from each of the four M. floribunda 821 trees. The fresh lesions were cut, placed into a beaker containing distilled water and $0.5 \mu 1 / \mathrm{ml}$ Tween 20, and shaken for $20 \mathrm{~min}$. The suspension was filtered by two layers of sterile cheesecloth, and the conidia concentration was estimated by a hemocytometer. To set the concentration to at least $3 \times 10^{4}$ conidia/ml, the suspension was centrifuged at $3,000 \mathrm{rpm}$ for $10 \mathrm{~min}$, and the supernatant was discarded to retain the precipitate. The resulting precipitate was resuspended in a minimal amount of distilled water and the final concentration was measured by hemocytometer.

Four 'Macfree' scions were grafted on 'M9' rootstocks and maintained in a greenhouse under controlled conditions, where the 1-yearold grafted plants were pruned to acquire new growth and young leaves. The spore inoculum was used to spray the first 4 to 6 actively growing intact leaves from the top. The inoculated plants were held in a moist chamber under $18^{\circ} \mathrm{C}$ and 12 -h photoperiod. To maintain $100 \%$ humidity, the sprayed leaves were covered first by wet paper towels, and then by resealable plastic bags. The incubated shoot part was tagged by marking the shoots below the bags to ease future evaluation. After $48 \mathrm{~h}$ of incubation, the bags were removed, and the plants were further maintained under low light, $18^{\circ} \mathrm{C}$ temperature, and $70 \%$ humidity with no irrigation for 2-3 weeks (Peil et al. 2018). As for the field assessment, reaction types were classed using the PRI program (Crosby et al. 1992). Light microscopy was used to evaluate the intensity of sporulation on the leaves. Spores from the infected leaves were suspended in a minimal amount of water and their morphology was observed under the microscope to confirm the identity of $V$. inaequalis. Images were taken using Toupview software with a digital camera for microscope (OMAX, Gyeonggi-do, South Korea).

DNA extraction and genome sequencing of $V$. inaequalis isolates. DNA was extracted from eight $V$. inaequalis monospore isolates, six of which were obtained from $M$. floribunda 821 , one from 'Golden Delicious', and one from 'Nova Easygro'. In case of M. floribunda 821, one, two, or three samples were collected from different parts of each of three different trees. In addition, DNA was extracted from two European isolates, EU-NL24 and EU-B05, and U.S. isolate VI-1797-9 from Purdue University (Table 2). Cultures were grown on potato dextrose agar for 4 weeks. No more than $0.2 \mathrm{~g}$ wet mycelia was cut and cleared from the agar by a sterile scalpel. Samples were ground in Eppendorf tubes with a disposable homogenizer pestle under liquid nitrogen. DNA was isolated with the Wizard Genomic DNA Purification Kit (Promega) as described by Singh and Khan (2019). The quality of DNA was checked using agarose gel (1\%) electrophoresis and quantified with Nanodrop and UV-Vis Spectrophotometer (Thermo Fisher Scientific, U.S.A.).

A total of $50 \mathrm{ng}$ DNA for each sample was used to prepare genome sequencing libraries for Illumina Nextera skim sequencing at Institute of Biotechnology, Cornell University, Ithaca, NY. Agilent Bioanalyzer (Agilent; https://www.agilent.com) was used to assess the quality and quantity of genomic libraries. Individual $V$. inaequalis samples were barcoded and sequenced in a single lane of Illumina Mi-Seq platform to generate $2 \times 250$ base pair paired-end read data.

Sequence analysis and variant detection. Raw sequences were separated into sample-specific reads with the barcode information and used to assess the sequence quality with fastqc program (http:// www.bioinformatics.babraham.ac.uk/projects/fastqc/). The program Trimmomatic (Bolger et al. 2014) was used to remove the sequencing adaptors and low-quality reads with quality scores below 20 . The genome assembly of $V$. inaequalis strain EU-B04 VeinUTG001 (Le Cam et al. 2019) was retrieved from the National Center for Biotechnology Information (NCBI) genome database and used to align the high-quality reads from the previous step. The alignments were filtered to eliminate PCR duplicated sequences and a sorted binary alignment format (BAM) was obtained for each sample using SAMtools ( $\mathrm{Li}$ et al. 2009).

Variants across the $V$. inaequalis genome were identified using Genome Analysis Toolkit (GATK version 3.8.0; McKenna et al. 2010) with sample-specific BAM files as input. The HaplotypeCaller plugin in GATK was used to obtain genotype variant call format ( $\mathrm{gVCF}$ ), and individual sample gVCF files were merged into a single variant file with the GenotypeGVCF plugin. The raw variants were processed through "hard filtering" criteria as per GATK best practices. The resulting variants were used for base recalibration to remove false positives. The variant dataset was separated into single nucleotide polymorphisms (SNPs) and insertions/deletions (INDELs).

Genetic diversity and population structure analysis. Genetic diversity and population genetic analysis was conducted with SNP data only. The SNPs were filtered using the minimum read depth criterion of four. Furthermore, we kept SNPs with no missing data even in a single sample. Remaining high-quality SNPs were used to calculate the genome-wide statistics of diversity, population structure, and selection indices in $V$. inaequalis. The population level nucleotide variation was examined through the number of variants, nucleotide diversity $(\pi)$, and principal component analysis (PCA) in Tassel v5 (Bradbury et al. 2007). The structure in the V. inaequalis population was further examined using the BYM admixture model and the haploid genome option in the TESS software (Caye et al. 2016) with default settings. In addition, spatial coordinates were provided for the 11 isolates to infer the accurate structure in $V$. inaequalis isolates. Convergence in the population structure results was checked by running 20 independent runs for individual $\mathrm{K}$ values ranging from two to eight. DIC and Kmax were plotted to identify the number of clusters/groups in the samples. Figures showing assignment of isolates to different clusters were generated

Table 2. The single spore isolates of Venturia inaequalis originating from Europe and Darrow farm (U.S.A.) from Malus floribunda 821, and apple cultivars 'Golden Delicious' and 'Nova Easygro'

\begin{tabular}{lllll}
\hline Code & \multicolumn{1}{c}{ Origin } & \multicolumn{1}{c}{ Host } & Host R genes & Reference \\
\hline VI-NY01-NE & New York, U.S.A. & Nova Easygro & Rvi6 & - \\
VI-NY02-MFa & New York, U.S.A. & M. floribunda 821 & Rvi6, Rvi7 & - \\
VI-NY02-MFb & New York, U.S.A. & M. floribunda 821 & Rvi6, Rvi7 & - \\
VI-NY02-MFc & New York, U.S.A. & M. floribunda 821 & Rvi6, Rvi7 & - \\
VI-NY03-MF & New York, U.S.A. & M. floribunda 821 & Rvi6, Rvi7 & - \\
VI-NY04-MFa & New York, U.S.A. & M. floribunda 821 & Rvi6, Rvi7 & - \\
VI-NY04-MFb & New York, U.S.A. & M. floribunda 821 & Rvil & - \\
VI-NY05-GD & New York, U.S.A. & Golden Delicious & Rvi4 & Schnabel et al. 1999 \\
VI-1797-9 & Ohio, U.S.A. & TSR33T239 & Rvil, Rvi6 & Caffier et al. 2015 \\
EU-NL24 & The Netherlands & Prima & None & Caffier et al. 2015 \\
EU-B05 & Belgium & Schone van Boskoop & & \\
\hline
\end{tabular}


using Clumpak - Cluster Markov Packager Across K (Kopelman et al. 2015).

\section{Results}

Apple scab infection in the orchard and under controlled conditions. In the beginning of June, scab symptoms were observed on M. floribunda 821 in the orchard at Darrow Farm, Geneva, NY on each of the four replicated trees. The initial sparse symptoms spread to approximately $10 \%$ (incidence grade 4 ) of the leaves later in July and August (Table 1). The heavy infection resulted in abundant sporulation (class 4), which often covered the whole leaf surface area (Fig. 1). Chlorosis and pin point pit type resistance reactions typical of the Rvi6 and Rvi7 genes, respectively, which are hypothetically the result of the large amount of inoculum from avirulent strains, were also frequent on the leaves.

The scab-susceptible commercial cultivars, 'Gala', 'Wijcik McIntosh', 'Marshall McIntosh', and 'Golden Delicious', all showed severe scab symptoms (class 4 ). Scab symptoms were more severe on 'Golden Delicious' than on 'Gala' (incidence 7 and 5, respectively). Many Rvi6 cultivars originating from M. floribunda 821, such as 'Dayton', 'Florina', 'Jonafree', 'Priscilla', and 'Redfree', were totally free of scab during the whole season. Weak symptoms were also detected on the descendants of M. floribunda 821, 'Prima', and 'Nova Easygro'; however, on these cultivars the infection was barely detectable. No more than one or two leaves per tree were infected in these cultivars (grade 1). In case of 'Nova Easygro', both chlorosis and necrosis were detected around the slight sporulation as the sign of an induced defense response (M), while the weak sporulating lesions on 'Prima' were all surrounded by unusually strong chlorosis (3).

Abundantly sporulating scab symptoms were observed under the microscope on the leaves of 'Macfree' 15 days after inoculation in controlled environment (Fig. 2). The sporulation covered a large part of the leaf surface area without any trace of a chlorotic or necrotic response, hence we consider this reaction to represent the full compatibility infection class (4).

Genomic diversity in the $\boldsymbol{V}$. inaequalis isolates. To understand the genetic diversity, population structure, and admixture, we sequenced and analyzed the $11 \mathrm{~V}$. inaequalis genomes in this study (Table 2). A total of 34.5 million raw sequencing reads were obtained, out of which $0.38 \%$ low quality reads were discarded from further analysis (Table 3 ). The filtered sequence data constituted about 3.1 million average reads per sample, and provided $9.2 \times$ coverage of the $V$. inaequalis genome in NCBI. The read alignment rate against the $V$. inaequalis genome varied from 97.7 to $99.2 \%$, with an average of $98.7 \%$ (Table 3 ).

After variant detection and GATK hard filtration, a total of 199,607 SNPs were identified with no missing data and representing an average of one SNP of every 360 base pairs of the $V$. inaequalis genome. About $38.1 \%(n=76,130)$ SNPs were detected in European isolates and $25.2 \%(n=50,292)$ were present in the two isolates collected from U.S. locations other than Darrow farm (Supplementary Fig. S1). The seven isolates from Darrow farm constituted about $68.3 \%(n=136,406)$ of the total SNPs identified. Approximately $4.4 \%$ of SNPs were shared among these three groups of $V$. inaequalis isolates (Supplementary Fig. S1). The six isolates from M. floribunda
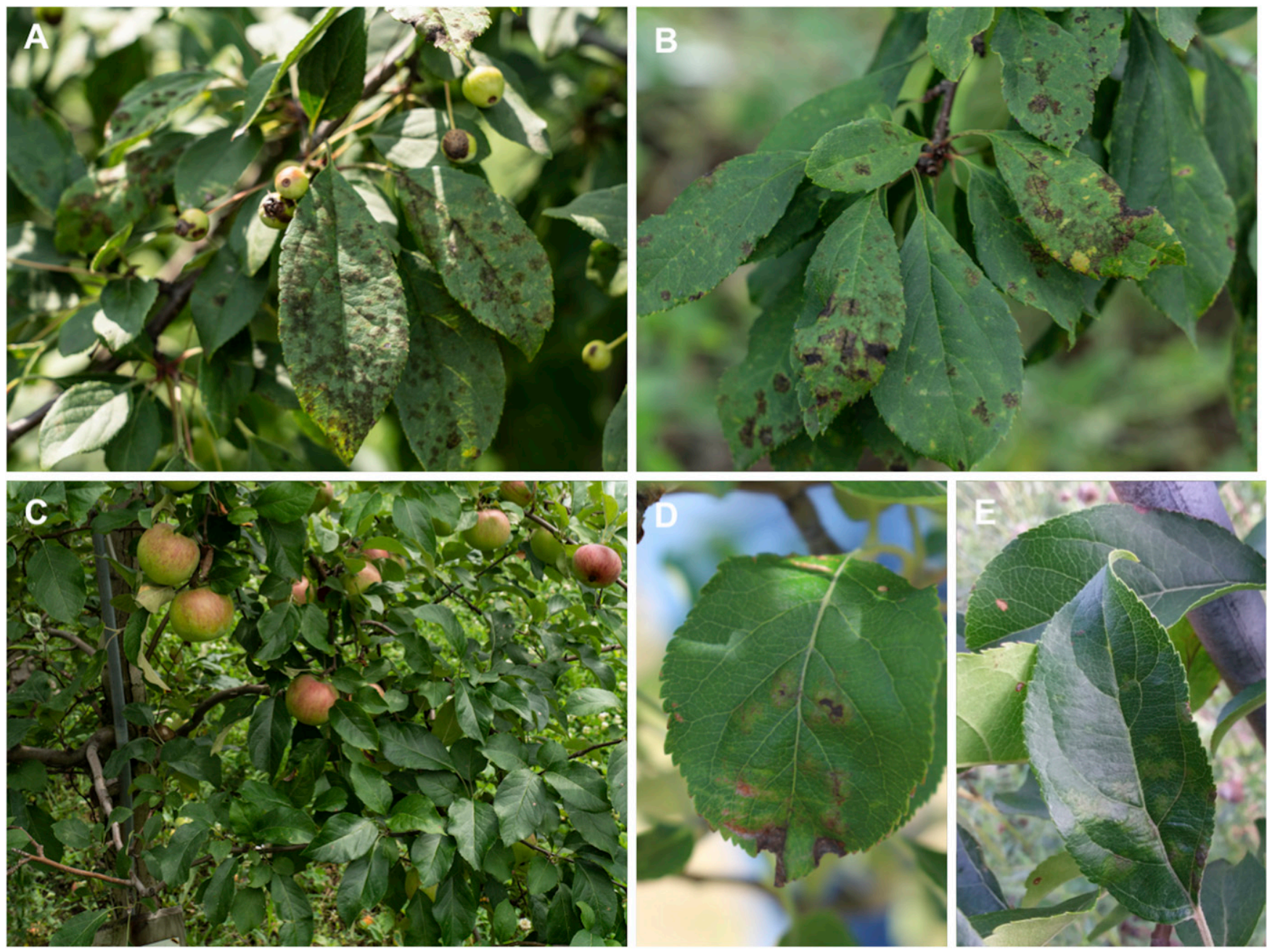

Fig. 1. Symptoms of apple scab infection detected on Malus floribunda 821, 'Nova Easygro' and 'Prima': heavy sporulation (A) alongside separate chlorotic and pin point type resistance symptoms (B) on M. floribunda 821; apparently intact crown of 'Nova Easygro' (C); weak sporulation surrounded by chlorosis and necrosis on 'Nova Easygro' (D) and by strong chlorosis on 'Prima' (E). 
821 had 119,109 SNPs, representing about $59.6 \%$ of the total genetic diversity in the population. There were 55,130 SNPs identified in the three isolates collected from the same $M$. floribunda 821 tree. We further observed about 0.29 nucleotide diversity $(\pi)$ in this $V$. inaequalis collection. The seven isolates collected from Darrow farm had a $\pi$ value of 0.39 and six isolates from $M$. floribunda 821 exhibited a $\pi$ value of 0.43 , thus representing slightly higher nucleotide diversity than the entire collection. The nucleotide diversity of the two U.S. isolates outside of Darrow farm and the two European isolates was not estimated due to limited sample size.

Population genetic structure analysis. The population structure was examined using 16,321 high-quality SNPs to perform principal component analysis (PCA) and BYM admixture estimation of $V$. inaequalis genomes. PCA clearly separated the 11 isolates into two distinct European and U.S. groups (Fig. 3). The two European isolates from Belgium and Netherlands also showed clear distinction from each other. The U.S. groups also exhibited a slightly dispersed pattern (Fig. 3). For instance, two $V$. inaequalis isolates from nonRvi6 hosts showed slightly distinct clustering from the five isolates from Rvi6 hosts, which exhibited much closer grouping among themselves on the PCA biplot. These results might reflect that host preference and isolate origin is in line with the genetic dissimilarities observed between these isolates. Furthermore, the remaining two isolates from Rvi6 hosts had a distinct placement in the PCA biplot. These two isolates represent two of the three samples that were collected from the same tree of $M$. floribunda 821, suggesting the existence of both clonal and nonclonal isolates on the same tree. The remaining isolate colocalized with the other isolates from $M$. floribunda 821. The other subgroup of 5 U.S. isolates from Darrow farm had a diverse host range including 'Golden Delicious', 'Nova Easygro', and M. floribunda 821.

A genome admixture analysis reflected four main clusters across the $11 \mathrm{~V}$. inaequalis isolates (Fig. 4). Two of the clusters represent the two European isolates and the other two clusters were specific to the U.S. isolates. A major proportion of genomic composition was different between the two European isolates, which also differed from the remaining isolates from the U.S. regions. A U.S. isolate VINY05-GD obtained from 'Golden Delicious' exhibited a partially different genomic composition from the other U.S. isolates from within and outside Darrow farm. The six isolates from $M$. floribunda 821 mostly showed similar genome composition except VI-NY03MF, which had some admixture from European genomes (Fig. 4). The main differences observed in genomic composition at $\mathrm{K}=4$ remained consistent as $K$ value changed from 3 to 5 . At $K=5$, the few U.S. isolates and two European isolates showed a small proportion of distinct genome other than the four groups observed at $\mathrm{K}=4$.

\section{Discussion}

We observed severe apple scab symptoms on trees of the Japanese crabapple $M$. floribunda 821 , which carries the scab resistance genes Rvi6 and Rvi7, in a research orchard at Geneva, NY in 2019. The breakdown of Rvi6 resistance was reported within a decade of its deployment from several European locations (Bénaouf and Parisi 1997; Parisi et al. 1993), but until now resistance has remained effective elsewhere. We have confirmed the loss of the resistance of $M$. floribunda 821 in North America. Seven cultivars descended from M. floribunda 821 ('Dayton', 'Jonafree', 'Prima', 'Priscilla', 'Redfree', 'Florina', and 'Nova Easygro') within the same orchard developed levels of scab much lower than observed on Japanese crabapple,

Table 3. Summary statistics of number of sequence reads generated, quality filtering, and percent alignment (mapping) against the Venturia inaequalis reference genome (Le Cam et al. 2019)

\begin{tabular}{lccc}
\hline Sample ID & Raw reads & Cleaned reads & Mapping (\%) \\
\hline VI-NY05-GD & 3549542 & 3549542 & 99.08 \\
VI-NY01-NE & 3223354 & 3223354 & 98.91 \\
VI-NY03-MF & 2850184 & 2850184 & 99.1 \\
VI-NY04-MFa & 3342636 & 3342636 & 99.2 \\
VI-NY04-MFb & 2453088 & 2453088 & 98.74 \\
VI-NY02-MFa & 3128934 & 3128934 & 98.93 \\
VI-NY02-MFb & 2770586 & 2770586 & 97.69 \\
VI-NY02-MFc & 2602972 & 2602972 & 99.27 \\
VI-1797-9 & 4555028 & 4509802 & 98.75 \\
EU-NL24 & 3015184 & 2973782 & 98.41 \\
EU-B05 & 2990158 & 2945712 & 98.54 \\
Total & 34481666 & 34350592 & 98.7 \\
Coverage & & 9.21 & \\
\hline
\end{tabular}

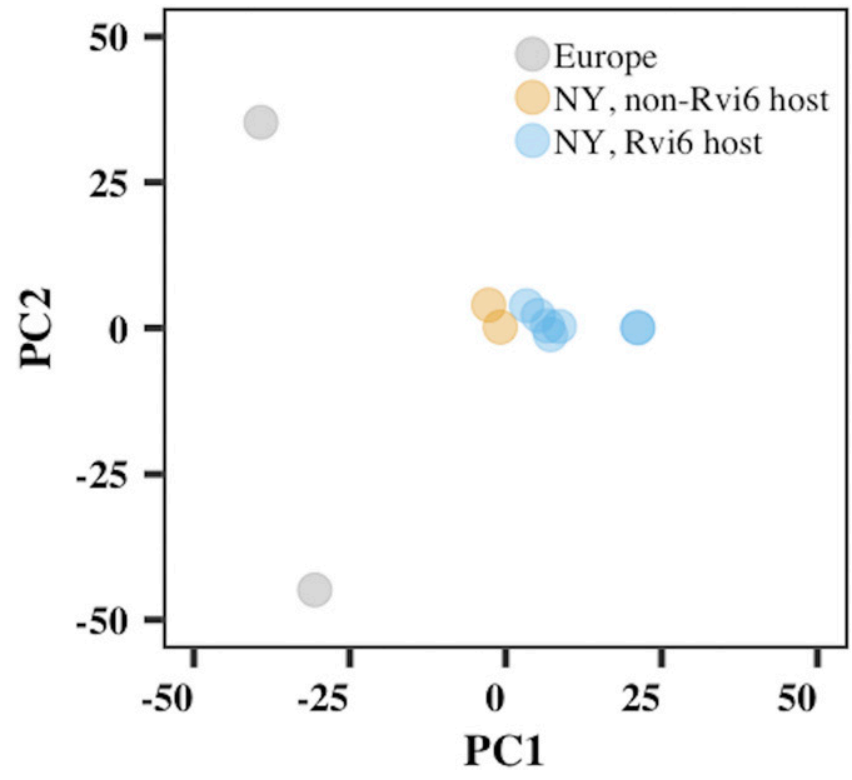

Fig. 3. Principal Component Analysis of 11 Venturia inaequalis isolates using 16,321 SNPs supported by a minimum of four sequencing reads. The original variant dataset was filtered to retain SNPs, supported by minimum four sequencing reads. The color of points on PCA biplot represent different groups of isolates, as indicated. Overlapping of data points is shown by darker color.
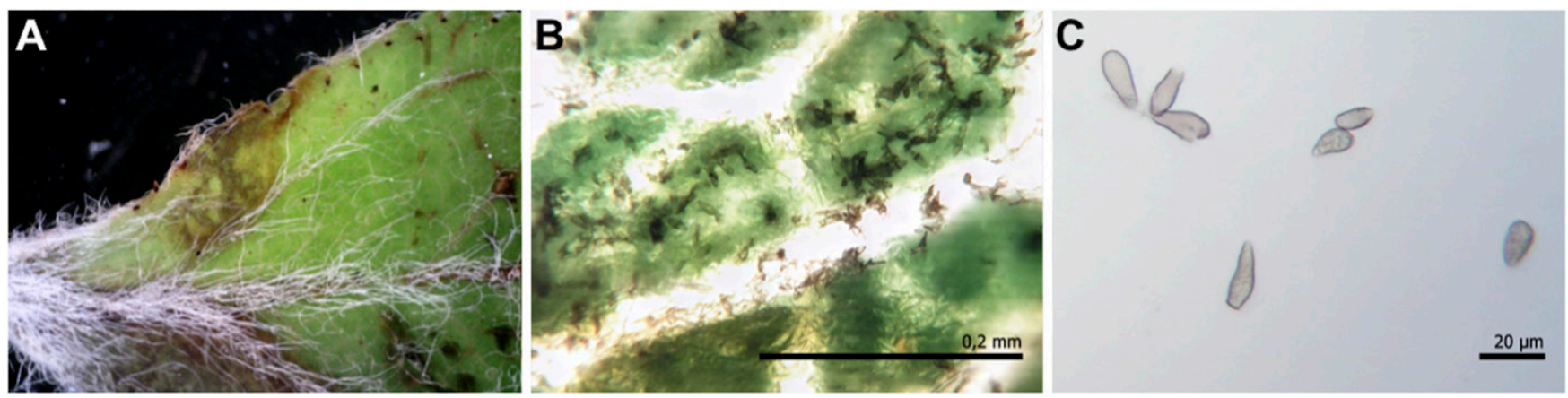

Fig. 2. Susceptible apple scab reaction of 'Macfree' (Rvi6) in a controlled environment to the inoculum of Venturia inaequalis isolates collected from the field from Malus floribunda 821. Sporulating leaf surface at $16 \times$ magnification $(\mathbf{A})$, macroscopic lesions (B), and suspended conidiospores at $40 \times$ magnification $(\mathbf{C})$. 
despite their common ancestry with Japanese crabapple and possession of Rvi6 (Table 1). The high incidence of scab across a range of known susceptible cultivars, and the uniform infection among all replicate trees of $M$. floribunda 821 , would indicate that low scab incidence among these remaining resistant cultivars was not due to escape, but because of the presence of Rvi6 in a genetic background still contributing additional resistance to $V$. inaequalis. Yet unidentified $R$ genes deriving from cultivars in the pedigree of $R v i 6$ cultivars might also contribute to their resistance. The foregoing would be consistent with the report by Roberts and Crute (1994) that many Rvi6 cultivars remained resistant to scab in Europe after the resistance of M. floribunda 821 had eroded there. Parisi et al. (2004) also reported that 'Prima' remained more resistant to scab than $M$. floribunda 821 when challenged by isolates able to overcome Rvi6. It has been proposed that Rvil, derived from 'Golden Delicious', or other ephemeral genes might contribute additional resistance when paired with Rvi6. We observed continued resistance to scab among many Rvi6 cultivars that to our knowledge do not carry Rvil (e.g., 'Priscilla' is used as differential host 6 as it is considered solely carrying Rvi6, Bus et al. 2011), meanwhile slight sporulation was observed on 'Prima' carrying Rvil alongside Rvi6 (Table 1). Gessler and Pertot (2012) speculated that Rvil might occasionally act indirectly against $R v i 6$ virulent isolates, and Caffier et al. (2010) hypothesized a fitness cost to virulence toward Rvi6 conferred by loss of avrRvi6. However, the ability to overcome a larger number of $\mathrm{R}$ genes (excluding Rvi6) has not correlated with low fitness in past studies (Parisi et al. 2004; Peil et al. 2018).

It is important to distinguish between mild chlorotic flecking typical of greenhouse inoculations and the loss of field resistance to apple scab, where profusely sporulating lesions are macroscopically visible in orchard assessments. The chlorotic and necrotic symptoms seen on the field on 'Prima' and 'Nova Easygro' cannot be considered as evidence that their field resistance has been overcome. Likewise, chlorotic lesions supporting sparse sporulation have been reported on 'Prima' following greenhouse inoculations (Crosby et al. 1992). Even in cases of compatible interactions between the host and the pathogen, from a practical standpoint, whether resistance breakdown can be considered significant might depend on the severity and economic impact (Delmotte et al. 2016). Sparse chlorotic sporulation on commercial Rvi6 cultivars is not generally considered a sign of resistance breakdown (Bus et al. 2011). On the other hand, Rvi6 cultivars including 'Macfree' often appear to be susceptible under greenhouse conditions, indicating the breakdown of Rvi6 resistance, but remains highly resistant in field plantings (Crosby et al. 1992; Roberts and Crute 1994). We observed similar mild and chlorotic symptoms on several scab resistant cultivars in the field (Table 1), but these are easily distinguished both qualitatively and quantitatively from the symptoms recorded on $M$. floribunda 821 .

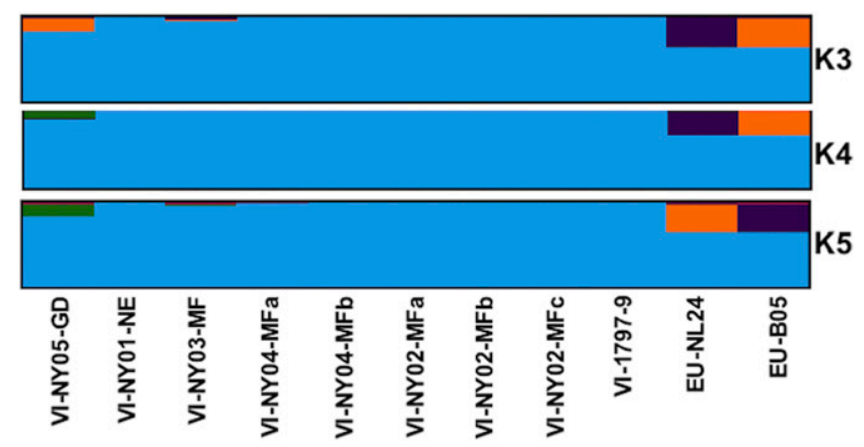

Fig. 4. Plot showing population structure and admixture levels in the genomes of 11 Venturia inaequalis isolates using 16,321 SNPs supported by minimum four sequencing reads. The analysis was conducted with the TESS software (Caye et al. 2016) using BYM admixture model and haploid genome with default settings. Convergence in the population structure results was checked by running 20 independent runs for individual $\mathrm{K}$ values. The best population structure was represented by $\mathrm{K}=4$.
The earlier discovery of Rvi6- and Rvi7-virulent isolates in Europe creates the possibility of transportation of such isolates to North America and subsequent spread. However, our very limited comparison of genetic polymorphism of the European and American virulent isolates suggested that both evolved or were selected from genetically distinct and separate populations. In Europe, the Rvi6-virulent lineage has existed separately in nonagricultural areas for thousands of years (Lemaire et al. 2016), which could also be true for North America. While there is considerable genetic distinction between the isolate from 'Golden Delicious' and the new virulent isolates, we do not have sufficient data to entirely exclude the possibility of gene flow and human transportation of isolates.

The considerable genetic polymorphism among the $M$. floribunda 821 virulent American isolates makes them less likely to be clonal variants. Interbreeding between Rvi6 virulent and nonvirulent isolates has already been documented in mixed orchards (Michalecka et al. 2018). Even though our isolates were collected within an unsprayed experimental orchard comprised of several cultivars, the genetic dissimilarity detected between the groups suggests that intense hybridization has not yet occurred between the M. floribunda 821 virulent and avirulent isolates, although a larger number of isolates should be analyzed to draw a conclusion.

To successfully manage the disease resistance of cultivars, the distribution and evolutionary processes of pathogen isolates must be understood. Despite the breakdown of resistance in M. floribunda 821, many of its descendants were uninfected in the field, or showed a scarcely noticeable amount of sporulation, hence remained field resistant. This suggests that the overall genetic background into which a single major $R$ gene is incorporated can substantially affect outcomes with respect to durability of resistance over time. Pyramiding major $R$ genes or involving partial polygenic resistance is considered a durable alternative to utilizing single major resistance genes (Corwin and Kliebenstein 2017; Khan and Chao 2017; Robinson 1996). Breeders prefer to choose the most promising disease resistance genes and gene combinations to achieve commercially successful cultivars with durable disease resistance. However, due to the time needed to select and breed for polygenic resistance and the possibility of linkage drag of undesirable alleles, in the past breeding programs have prioritized major monogenic resistances. In-depth understanding of the factors resulting in the emergence of M. floribunda 821 resistance-breaking isolates, and the breakdown of resistance from M. floribunda 821 but not in descendant cultivars, may ultimately reduce the risk of eroding the resistance of Rvi6 cultivars and enhance their durability.

\section{Acknowledgments}

We thank David Strickland and Liqiang Gao for culturing and DNA extraction of some of the isolates used in the study and Della Cobb-Smith for technical support.

\section{Literature Cited}

Beckerman, J., Chatfield, J., and Draper, E. 2009. A 33-year evaluation of resistance and pathogenicity in the apple scab - crabapples pathosystem. HortScience 44:599-608.

Beckerman, J. L., Sundin, G. W., and Rosenberger, D. A. 2015. Do some IPM concepts contribute to the development of fungicide resistance? Lessons learned from the apple scab pathosystem in the United States. Pest Manag. Sci. 71:331-342.

Bénaouf, G., and Parisi, L. 1997. Pathogenicity of Venturia inaequalis strains from Malus floribunda 821: Comparison with race 6 on apple clones. IOBC WPRS Bull. 20:8-11.

Bénaouf, G., and Parisi, L. 2000. Genetics of host-pathogen relationships between Venturia inaequalis races 6 and 7 and Malus species. Phytopathology 90: 236-242.

Bolger, A. M., Lohse, M., and Usadel, B. 2014. Trimmomatic: A flexible trimmer for Illumina sequence data. Bioinformatics 30:2114-2120.

Bradbury, P. J., Zhang, Z., Kroon, D. E., Casstevens, T. M., Ramdoss, Y., and Buckler, E. S. 2007. TASSEL: Software for association mapping of complex traits in diverse samples. Bioinformatics 23:2633-2635.

Broggini, G. A., Bus, V. G., Parravicini, G., Kumar, S., Groenwold, R., and Gessler, C. 2011. Genetic mapping of 14 avirulence genes in an EU-B04 $\times$ 1639 progeny of Venturia inaequalis. Fungal Genet. Biol. 48:166-176.

Brown, S., and Maloney, K. 2008. Scab-resistant cultivars (varieties). N.Y. Fruit Q. 16:3-6. 
Brown, S. K., and Maloney, K. E. 2013. An update on apple cultivars, brands and club-marketing. N.Y. Fruit Q. 21:3-10.

Bus, V. G., Rikkerink, E. H., Caffier, V., Durel, C. E., and Plummer, K. M. 2011. Revision of the nomenclature of the differential host-pathogen interactions of Venturia inaequalis and Malus. Annu. Rev. Phytopathol. 49:391-413.

Caffier, V., Didelot, F., Pumo, B., Causeur, D., Durel, C. E., and Parisi, L. 2010. Aggressiveness of eight Venturia inaequalis isolates virulent or avirulent to the major resistance gene Rvi6 on a non-Rvi6 apple cultivar. Plant Pathol. 59: 1072-1080.

Caffier, V., Patocchi, A., Expert, P., Bellanger, M. N., Durel, C. E., HilberBodmer, M., Broggini, G. A. L., Groenwold, R., and Bus, V. G. 2015. Virulence characterization of Venturia inaequalis reference isolates on the differential set of Malus hosts. Plant Dis. 99:370-375.

Caye, K., Deist, T. M., Martins, H., Michel, O., and François, O. 2016. TESS3: Fast inference of spatial population structure and genome scans for selection. Mol. Ecol. Resour. 16:540-548.

Chapman, K. S., Sundin, G. W., and Beckerman, J. L. 2011. Identification of resistance to multiple fungicides in field populations of Venturia inaequalis. Plant Dis. 95:921-926.

Corwin, J. A., and Kliebenstein, D. J. 2017. Quantitative resistance: More than just perception of a pathogen. Plant Cell 29:655-665.

Crandall, C. S. 1926. Apple breeding at the University of Illinois. Ill. Agric. Exp. Stn. Bull. 275:341-600.

Crosby, J. A., Janick, J., Pecknold, P. C., Korban, S. S., O’Connor, P. A., Ries, S. M., Goffreda, J., and Voordeckers, A. 1992. Breeding apples for scab resistance: 1945-1990. Acta Hortic.: 43-70.

Delmotte, F., Bourguet, D., Franck, P., Guillemaud, T., Reboud, X., Vacher, C., and Walker, A. S. 2016. Combining selective pressures to enhance the durability of disease resistance genes. Front. Plant Sci. 7:1916.

Gao, Z. S., and Van De Weg, W. E. 2006. The Vf gene for scab resistance in apple is linked to sub-lethal genes. Euphytica 151:123-132.

Gessler, C. 1989. Genetics of the interaction Venturia inaequalis-Malus: The conflict between theory and reality. IOBC Bull XII:168-190.

Gessler, C., and Pertot, I. 2012. Vf scab resistance of Malus. Trees (Berl.) 26: 95-108.

Gladieux, P., Zhang, X. G., Róland-Ruiz, I., Caffier, V., Leroy, T., Devaux, M., Van Glabeke, S., Coart, E., and Le Cam, B. 2010. Evolution of the population structure of Venturia inaequalis, the apple scab fungus, associated with the domestication of its host. Mol. Ecol. 19:658-674.

Guérin, F., Gladieux, P., and Le Cam, B. 2007. Origin and colonization history of newly virulent strains of the phytopathogenic fungus Venturia inaequalis. Fungal Genet. Biol. 44:284-292.

Guérin, F., and Le Cam, B. 2004. Breakdown of the scab resistance gene Vf in apple leads to a founder effect in populations of the fungal pathogen Venturia inaequalis. Phytopathology 94:364-369.

Kaymak, S., Boyraz, N., and Daniels, J. 2016. Molecular markers to evaluate genetic diversity among Venturia inaequalis isolates obtained from apple plantations in Isparta Province. Turk. J. Agric. For. 40:489-498.

Khajuria, Y. P., Kaul, S., Wani, A. A., and Dhar, M. K. 2018. Genetics of resistance in apple against Venturia inaequalis (Wint.) Cke. Tree Genet. Genomes 14:16.

Khan, M. A., and Chao, T. 2017. Wild apple species as a source of fire blight resistance for sustainable productivity of apple orchards. N.Y. Fruit Q. 25:13-18.
Kopelman, N. M., Mayzel, J., Jakobsson, M., Rosenberg, N. A., and Mayrose, I. 2015. Clumpak: A program for identifying clustering modes and packaging population structure inferences across K. Mol. Ecol. Resour. 15:1179-1191.

Lateur, M., and Populer, C. 1994. Screening fruit tree genetic resources in Belgium for disease resistance and other desirable characters. Euphytica 77:147-153.

Le Cam, B., Sargent, D., Gouzy, J., Amselem, J., Bellanger, M. N., Bouchez, O., Brown, S., Caffier, V., De Gracia, M., Debuchy, R., Duvaux, L., Payen, T., Sannier, M., Shiller, J., Collemare, J., and Lemaire, C. 2019. Population genome sequencing of the scab fungal species Venturia inaequalis, Venturia pirina, Venturia aucupariae and Venturia asperata. G3: Genes, Genomes. Genetics 9:2405-2414.

Lemaire, C., De Gracia, M., Leroy, T., Michalecka, M., Lindhard-Pedersen, H. Guerin, F., Gladieux, P., and Le Cam, B. 2016. Emergence of new virulent populations of apple scab from nonagricultural disease reservoirs. New Phytol. 209:1220-1229.

Li, H., Handsaker, B., Wysoker, A., Fennell, T., Ruan, J., Homer, N., Marth, G., Abecasis, G., and Durbin, R. 2009. The sequence alignment/map format and SAMtools. Bioinformatics 25:2078-2079.

MacHardy, W. E. 1996. Apple Scab: Biology, Epidemiology, and Management. American Phytopathological Society, St. Paul, MN.

McKenna, A., Hanna, M., Banks, E., Sivachenko, A., Cibulskis, K., Kernytsky, A. Garimella, K., Altsuler, D., Gabriel, S., Daly, M., and DePristo, M. A. 2010. The Genome Analysis Toolkit: A MapReduce framework for analyzing nextgeneration DNA sequencing data. Genome Res. 20:1297-1303.

Michalecka, M., Masny, S., Leroy, T., and Puławska, J. 2018. Population structure of Venturia inaequalis, a causal agent of apple scab, in response to heterogeneous apple tree cultivation. BMC Evol. Biol. 18:5.

Parisi, L., Fouillet, V., Schouten, H. J., Groenwold, R., Laurens, F., Didelot, F., Evans, K., Fischer, C., Gennari, F., Kemp, H., Lateur, M., Patocchi, A., Thissen, J., and Tsipouridis, C. 2004. Variability of the pathogenicity of Venturia inaequalis in Europe. Acta Hortic. 663:107-113.

Parisi, L., and Lespinasse, Y. 1996. Pathogenicity of Venturia inaequalis strains of race 6 on apple clones (Malus sp.). Plant Dis. 80:1179-1183.

Parisi, L., Lespinasse, Y., Guillaumes, J., and Krüger, J. 1993. A new race of Venturia inaequalis virulent to apples with resistance due to the $V f$ gene. Phytopathology 83:533-537.

Peil, A., Patocchi, A., Hanke, M. V., and Bus, V. G. 2018. Apple cultivar Regia possessing both Rvi2 and Rvi4 resistance genes is the source of a new race of Venturia inaequalis. Eur. J. Plant Pathol. 151:533-539.

Pink, D. A. C., and Hand, P. 2003. Plant resistance and strategies for breeding resistant varieties. Plant Prot. Sci. 38 (SI 1 - 6th Conf EFPP 2002):S9-S14.

Roberts, A. L., and Crute, I. R. 1994. Apple scab resistance from Malus floribunda $821(\mathrm{~V} f)$ is rendered ineffective by isolates of Venturia inaequalis from Malus floribunda. Nor. J. Agric. Sci. 17:403-406.

Robinson, R. A. 1996. Return to resistance. Breeding Crops to Reduce Pesticide Dependence. Agaches, Davis, CA.

Schnabel, G., Schnabel, E. L., and Jones, A. L. 1999. Characterization of ribosomal DNA from Venturia inaequalis and its phylogenetic relationship to rDNA from other tree-fruit Venturia species. Phytopathology 89:100-108.

Singh, J., and Khan, A. 2019. Distinct patterns of natural selection determine subpopulation structure in the fire blight pathogen, Erwinia amylovora. Sci. Rep. 9:14017.

Williams, E. B., and Kuc, J. 1969. Resistance in Malus to Venturia inaequalis. Annu. Rev. Phytopathol. 7:223-246. 